\title{
Abstract: Deep Learning Based CT-CBCT Image Registration for Adaptive Radio Therapy
}

\author{
Sven Kuckertz ${ }^{1}$, Nils Papenberg ${ }^{1}$, Jonas Honegger ${ }^{2}$, Tomasz Morgas ${ }^{2}$, \\ Benjamin Haas ${ }^{2}$, Stefan Heldmann ${ }^{1}$ \\ ${ }^{1}$ Fraunhofer Institute for Digital Medicine MEVIS, Lübeck, Germany \\ ${ }^{2}$ Varian Medical Systems, Baden-Dättwil, Switzerland \\ sven.kuckertz@mevis.fraunhofer.de
}

Deformable image registration (DIR) is an important tool in radio therapy where it is used in order to align a baseline CT and daily low-dose cone beam CT (CBCT) scans. DIR allows the propagation of irradiation plans, Hounsfield units and contours of anatomical structures, respectively, which enables tracking of applied doses over time and generation of daily synthetic CT images. Furthermore, DIR allows to overcome segmentation of structures in CBCT images at each fraction. We present a novel weakly supervised deep learning based method for fast deformable registration of 3D images [1], targeting the challenges of multi-modal CT-CBCT registration that goes along with low contrast and artifacts in CBCT scans and extreme deformations of organs such as bladder.

The parameters of our convolutional neural network (CNN) are adapted for minimizing a loss function inspired from state-of-the-art iterative image registration. Therefore, our training is unsupervised and does not require any hard to obtain ground-truth deformation vector fields. Additionally, we include a weak supervision through ground-truth segmentations during training. We evaluate our proposed method on follow-up image pairs of the female pelvis including outlines of the bladder, rectum and uterus, showing that incorporating a measure for segmentation mask overlap during training enhances the alignment of organs with extreme deformations. Hence, our method outperforms state-of-the-art iterative DIR algorithms in terms of Dice overlap (in average 0.78 vs. 0.71) and average surface distance $(3.10 \mathrm{~mm}$ vs. $4.17 \mathrm{~mm})$, where both methods only use the image pair as input. Furthermore, it is nearly as good as iterative structure guided registration that depends on ground-truth segmentations, which first have to be generated after CBCT acquisition. Needing only one pass through the network, our method yields deformations over 100 times faster than conventional iterative algorithms $(0.13 \mathrm{~s}$ vs. $15.39 \mathrm{~s})$. Not requiring any segmentations of unseen image pairs, our framework additionally accelerates and facilitates the workflow of adaptive radio therapy.

\section{References}

1. Kuckertz S, Papenberg N, Honegger J, et al. Deep learning based CT-CBCT image registration for adaptive radio therapy. Proc SPIE. 2020;. 